\title{
EFFECTS OF SEGREGATION OF MARKED AND NORMAL SPECIES IN SANS FROM CRYSTALLINE POLYMERS
}

\author{
G.C. SUMMERFIELD \\ Department of Nuclear Engineering, The University of Michigan, Ann Arbor, MI 48109, USA
}

\begin{abstract}
The use of deuterium marking has proved to be very valuable in studying single-chain behavior in amorphous polymer systems. However, it has been difficult to use the technique for crystalline polymers because of anomalous forward scattering. This scattering has been attributed to segregation of the normal and deuterated species resulting from the small differences between them. Computations of the effects of segregtion have all assumed the absence of correlation between concentration and density fluctuations. This assumption precludes the possibility of different concentrations of marked polymer in the amorphous and crystalline regions of the sample. To explain the magnitudes of the anomalous scattering the previous computations required what seems to be extreme segregation of the two species. Here we compute the effects of concentration variations between the amorphous and crystalline regions and show that even modest differences in concentration can produce the observed magnitudes of the anomalous, forward scattering. Unfortunately the results seem to show that single chain SANS experiments in segregated, crystaline polymers are fundamentally problematical.
\end{abstract}

\section{Introduction}

The study of single chain characterisics of amorphous polymers using SANS on samples composed of mixtures of normal and deuterated polymer has contributed greatly to our undrstanding of dense polymer systems. However, attempts [1-5] to apply the technique to crystalline polymers have been plagued by anomalous, forward scattering. This anomalous, forward scattering has been attributed by all authors to some form of segregation of the deuterated and normal species beause of the small differences between them [1-5].

There have been attempts to calculate the magnitude of this excess scattering [1-5]. In each calculation, the author assumed some model for the manner in which the species segregated. It seems fair to say that, in every case, an extreme degree of segregation was needed to explain the magnitude of the excess scattering. A common feature of all of these models is the absence of a correlation between concentration and density fluctions. That is, there is assumed to be no correlation between regions of high or low concentration of marked polymer and regions of high or low polymer density. If the sample is composed only of crystalline and amorphous regions, this would mean that the overall concentration of marked polymer in these two regions would be the same. This assumption was tested in ref. 3. It also would mean that the patterns of segregaion in the two regions would be the same. These do not seem to be entirely reasonable assumptions. Here we shall try to rectify the assumption of an absence of correlation between density and concentration fluctions. We shall assume that the concentrations of deuterated polymer in the amorphous and crystalline regions are different.

\section{Calculations}

The derivation of the neutron scattering from a mixture of normal and deuterated polymer can be written in terms of a set of probabilities $P_{\mathrm{NHN}^{\prime} \mathrm{H}}$, $P_{\mathrm{NDN}^{\prime} \mathrm{D}}$ and $P_{\mathrm{NDN}^{\prime} \mathrm{H}}[6]$, where, for example, $P_{\text {NHN'H }}$ is the probability that the polymers whose centers of mass are at $\boldsymbol{R}_{\mathrm{N}}$ and $\boldsymbol{R}_{\mathrm{N}^{\prime}}$ are both normal. Here we assume that this probability depends upon whether $\boldsymbol{R}_{\mathrm{N}}$ and $\boldsymbol{R}_{\mathrm{N}}$, are in an amorphous or crystalline region. So, let us label chains as $\mathbf{N}$ or $\mathbf{M}$ if the center of mass is in a crystalline or amorphous region respectively. If 
we take the monomer positions relative to the center of mass to be $r_{l}$ for crystalline and $\boldsymbol{r}_{i}$ for amorphous regions, we can write the neutron cross section as

$$
\begin{aligned}
S(\boldsymbol{Q})= & \langle| \sum_{\mathrm{M}, i} a_{\mathrm{M}} \exp \left(\mathrm{i} \boldsymbol{Q} \cdot\left(\boldsymbol{R}_{\mathrm{M}}+\boldsymbol{r}_{i}\right)\right) \\
& \left.+\left.\sum_{\mathrm{N}, l} a_{\mathrm{N}} \exp \left(\mathrm{i} \boldsymbol{Q} \cdot\left(\boldsymbol{R}_{\mathrm{N}}+\boldsymbol{r}_{l}\right)\right)\right|^{2}\right\rangle .
\end{aligned}
$$

Here $a_{\mathrm{M}}$ and $a_{\mathrm{N}}$ are the scattering lengths of the monomers, $Q$ is the neutron wave vector transfer, and $\langle\cdots\rangle$ signifies the ensemble average.

We now need a larger set of probabilities since we assume that they depend upon where the centers of mass of the polymers are. Our assumption is quite simple. It is that the relative concentrations of marked polymer are $x_{\mathrm{A}}$ and $x_{\mathrm{C}}$ in the amorphous and crystalline regions respectively. Other than this, we assume that the mixing of the species is random. Now we need ten probabilities. Six of them, $P_{\mathrm{MHM}^{\prime} \mathrm{H}}, P_{\mathrm{MDM}^{\prime} \mathrm{D}}, P_{\mathrm{MHM}^{\prime} \mathrm{D}}, P_{\mathrm{NHN}^{\prime} \mathrm{H}}$, $P_{\mathrm{NDN} N^{\prime}}$, and $P_{\mathrm{NHN} \text { D }}$, the same as given in reference [6] with the appropriate $x\left(x_{\mathrm{C}}\right.$ or $\left.x_{\mathrm{A}}\right)$. The other four are $P_{\mathrm{MHNH}}=\left(1-x_{\mathrm{A}}\right)\left(1-x_{\mathrm{C}}\right)$, $P_{\text {MDND }}=x_{\mathrm{A}} x_{\mathrm{C}}, \quad P_{\mathrm{MHND}}=\left(1-x_{\mathrm{A}}\right) x_{\mathrm{C}}, \quad$ and $P_{\mathrm{MDNH}}=x_{\mathrm{A}}\left(1-x_{\mathrm{C}}\right)$. Using these $P$ 's, we obtain for equation (1)

$$
\begin{aligned}
S(\boldsymbol{Q})= & \left(a_{\mathrm{H}}-a_{\mathrm{D}}\right)^{2} n^{2}\left\{x_{\mathrm{C}}\left(1-x_{\mathrm{C}}\right) N_{\mathrm{C}} S_{\mathrm{sC}}(\boldsymbol{Q})\right. \\
& \left.+x_{\mathrm{A}}\left(1-x_{\mathrm{A}}\right) N_{\mathrm{A}} S_{\mathrm{sA}}(\boldsymbol{Q})\right\} \\
& +\langle| a_{\mathrm{A}} \sum_{\mathrm{M}, i} \exp \left(\mathrm{i} \boldsymbol{Q} \cdot\left(\boldsymbol{R}_{\mathrm{M}}+\boldsymbol{r}_{i}\right)\right) \\
& \left.+\left.a_{\mathrm{C}} \sum_{\mathrm{N}, l} \exp \left(\mathrm{i} \boldsymbol{Q} \cdot\left(\boldsymbol{R}_{\mathrm{N}}+\boldsymbol{r}_{l}\right)\right)\right|^{2}\right\rangle
\end{aligned}
$$

where $a_{\mathrm{C}}=a_{\mathrm{H}}\left(1-x_{\mathrm{C}}\right)+a_{\mathrm{D}} x_{\mathrm{C}}$ and $a_{\mathrm{A}}=a_{\mathrm{H}}(1-$ $\left.x_{\mathrm{A}}\right)+a_{\mathrm{D}} x_{\mathrm{D}} ; n$ is the degree of polymerization; $N_{\mathrm{A}}$ and $N_{\mathrm{C}}$ are the numbers of chains with centers of mass in the amorphous and crystalline regions respectively; and $S_{\mathrm{sC}}$ and $S_{\mathrm{sA}}$ are the single chain scattering functions,

$S_{\mathrm{sC}}(\boldsymbol{Q})=\left(1 / n^{2}\right)\left\langle\left|\sum_{l} \exp \left(\mathrm{i} \boldsymbol{Q} \cdot \boldsymbol{r}_{l}\right)\right|^{2}\right\rangle$,

$$
S_{\mathrm{sA}}(\boldsymbol{Q})=\left(1 / n^{2}\right)\left\langle\left|\sum_{i} s \exp \left(\mathrm{i} \boldsymbol{Q} \cdot \boldsymbol{r}_{i}\right)\right|^{2}\right\rangle .
$$

We cannot extract the single chain form factors from (2). We cannot even extract their average as given in the first term of (2) because of the complexity of the last term. The last term is not just a total scattering term since the amorphous and crystalline contributions are weighted differently.

In order to proceed, we must make some further assumption. It is not a simple matter to determine the last term in (2). However, there is one assumption that we can make which does simplify this term and which can give us an idea of its magnitude. This assumption is that chains remain in the same region as their centers of mass. Then the sum over $\mathrm{N}$ and $l$ is just a sum over the monomers in the crystalline regions and the sum over $\mathbf{M}$ and $i$ is just a sum over monomers in the amorphous regions. Then it is an easy matter to show that the last term in (2) becomes

$$
\left(a_{\mathrm{C}} \rho_{\mathrm{C}}-a_{\mathrm{A}} \rho_{\mathrm{A}}\right)^{2}\left|\int \mathrm{d}^{3} r \exp (\mathrm{i} Q \cdot r) \gamma(r)\right|^{2},
$$

where $\gamma$ is zero in amorphous regions and unity in crystalline regions.

\section{Discussion}

(5) is effectively the Debye-Bueche [7] result for scattering from a two component system in which the contrast of the components is $a_{\mathrm{C}} \rho_{\mathrm{C}}-$ $a_{\mathrm{A}} \rho_{\mathrm{A}}$. Even when $x_{\mathrm{A}}$ and $x_{\mathrm{C}}$ are quite close to the average concentration, this contrast can be very large and can produce a large forward scattering. In fact $\left(x_{\mathrm{A}}-x_{\mathrm{C}}\right) \approx 10^{-2}$ will produce forward scattering of the observed magnitude. We will not pursue this numerical computation further since the assumptions we made going from (2) to (5) are clearly not valid. The result does show that there can be a very large effect from having different concentrations of marked polymer in the crystalline and amorphous regions. Unfortunately (2) also shows that, in that case, we cannot extract single chain form factors. 


\section{Acknowledgement}

This work was supported by the National Science Foundation, Grant \#DMR 8217460.

\section{References}

[1] D.G.H. Ballard, A. Cunningham and J. Schelten, Polymer 18 (1977) 259.
[2] G.C. Summerfield, J.S. King and R. Ullman, J. Appl. Cryst. 11 (1978) 548.

[3] J. Schelten, D.G.H. Ballard, G. Wignall, G Longman and W. Schmatz, Polymer 17 (1976) 751.

[4] G. Allen and T. Tanaka Polymer 19 (1978) 271.

[5] D.M. Sadler and A. Keller, Macromolecules 10 (1977) 1128.

[6] A.Z. Akcasu, G.C. Summerfield, S.N. Jahshan, C.C. Han, C.Y. Kim and H. Yu, J. Polymer Sci., Polymer Phys. 18 (1980) 863.

[7] P. Debye and A.M. Bueche, J. Appl. Phys. 20 (1949) 518. 